\title{
Implant Placement in a Cemento-Osseous Dysplasia: A case report
}

\author{
Alexandre Perez, Avigaïl Maman, Edouard Di Donna, Tommaso Lombardi \\ Unit of Oral Medicine and Pathology, Division of Oral Maxillofacial Surgery, Department of Surgery, University Hospitals of \\ Geneva, Geneva, Switzerland
}

Received: June 21, 2021

Accepted: July 14, 2021

Online Published: August 7, 2021

DOI: $10.5430 /$ dcc.v8n2p6

URL: https://doi.org/10.5430/dcc.v8n2p6

\begin{abstract}
We present a 45-year-old black woman diagnosed with a florid cemento-osseous dysplasia (FCOD) affecting the right lower quadrant and the anterior mandibular region. The patient requested dental implants to rehabilitate the edentulous central lower incisors area (teeth \# 31 and \# 41) corresponding to a periapical cemento-osseous dysplasia (PCOD). Successful osseointegration of the two implants was obtained using a two-step procedure in order to limit the risk of complications associated with implant placement. Follow-up at one year showed no recurrence and good implant stability. Due to the abnormal quality of the bone in cemento-osseous dysplasia (COD), implant placement is generally avoided, and no other case reports have been reported in the literature in patients affected by PCOD. The present case suggests that in an appropriate clinical setting, implant placement may be a successful procedure.
\end{abstract}

Key Words: Cemento-Osseous Dysplasia, Periapical Cemento-Osseous Dysplasia, Dental implant

\section{INTRODUCTION}

Cemento-Osseous Dysplasia (COD) is a relatively frequent lesion characterized by the replacement of normal maxillary or mandibular bone by fibrous tissue containing cementumlike tissue. Those quantities depend on the stage of the disease. ${ }^{[1]}$

In 2017, the WHO described different clinical forms of COD: $:^{[2]}$

(1) The Periapical Cemento-Osseous Dysplasia (PCOD) in the periapical region of anterior mandibular teeth.

(2) Focal COD: generally, a single lesion in the posterior part of the jaw.

(3) Florid COD: with the multifocal distribution, lesion affecting both maxilla and mandible.
PCOD is the most frequent clinical form of COD. In a study including 118 patients with $\mathrm{COD},{ }^{[3]}$ the prevalence of PCOD was $78.8 \%$, and the incidence was $82.9 \%$ in females. It typically affects black middle-aged women, aged mostly between 30 and 50, rarely before 20 , and is almost always present in the periapical region of mandibular incisor. ${ }^{[4]}$

As suggested in a case report, PCOD may have a genetic component in addition to environmental factors with an inheritance autosomal dominant mode. ${ }^{[5]}$ The etiology remains unclear, but its origin from the periodontal ligament seems to be the most accepted hypothesis because of clinical and histological evidence. ${ }^{[6]}$

Three radiologic and histologic stages can be identified ${ }^{[4,7,8]}$ depending on the quantity of mineralized tissue:

* Correspondence: Alexandre Perez; Email: Alexandre.Perez@hcuge.ch; Address: Unit of Oral Medicine and Pathology, Division of Oral Maxillofacial Surgery, Department of Surgery, University Hospitals of Geneva, 1205 Geneva, Switzerland. 
First stage: osteolytic, characterized by radiolucent areas around apices of mandibular incisors.

Second stage: cementoblasts, in which the lesion appears partly radiopaque with calcifications in the center of the radiolucent lesion.

Third stage: a well-defined, dense, radiopaque lesion surrounded by a radiolucent rim.

Diagnosis is usually made on clinical and radiological features, the majority of cases PCOD being totally asymptomatic and therefore discovered on X-rays fortuitously. It occurs in the periapical region of vital teeth, rarely restored. Differential diagnoses of early-stage PCOD could include a periapical granuloma or a radicular cyst; in such cases, vitality tests are necessary to determine the difference. PCOD is usually asymptomatic, and therefore no treatment is indicated, but a follow-up of the lesion's evolution is recommended. ${ }^{[9,10]}$

Major complications that have been reported occurring in COD, such as secondary infections, are hardly controllable due to the low vascularity of the lesion site and, therefore, the inefficiency of antibiotics. ${ }^{[10]}$ In fact, trauma caused by tooth extraction, periodontal surgery, biopsy, or implant placement could lead to necrosis and osteomyelitis. ${ }^{[11]}$ For these reasons, dental implants are usually avoided in such a disease. Dental implant therapy has not been applied, to the best of our knowledge, in areas with PCOD.

We describe here a successful outcome in a patient who had implants placed in a PCOD.

\section{Case presentation}

A 45-year-old black woman in good general health presented at the Department of Maxillofacial Surgery, Unit of Oral Surgery, Geneva University Hospital with the chief complaint of functional and esthetic disturbance due to her missing teeth \# 31 and \# 41 extracted some years before due to generalized chronic periodontitis.

Periapical radiographs showed radiolucent and radiopaque lesions in the anterior mandibular region (see Figure 1), evocating a PCOD. The lesions were located in the periapical region of teeth \# 32 and \# 42 and manifested as a round, radiolucent, well-defined lesion of $6 \mathrm{~mm}$ in diameter close to the apex of tooth \# 42 (osteolytic stage) and as a radiopaque slightly larger lesion at the apex of tooth \# 32 corresponding to a later stage of the disease.

Radiolucent and radiopaque asymptomatic lesions were also discovered in the third quadrant in a periapical radiograph (see Figure 2). The radiological appearance of the lesions

Published by Sciedu Press and the location were consistent with an FCOD.

The patient requested implants therapy to replace the missing teeth in the anterior mandibular area. She was informed about the risks due to her condition. Two dental implants Straumann ${ }^{\circledR}$ BLT with a $2.9 \mathrm{~mm}$ in diameter and a $10 \mathrm{~mm}$ length, were placed at the edentulous sites. The two-stage dental implant surgery was chosen in order to avoid any infection by burying implants during the healing period. The apical portion of the implants was placed within the osseous lesion due to its proximity to the alveolar ridge. Follow-up showed good healing at the implant sites. A few months later, the bone loss occurred around the implants' necks and caused their exposition (see Figure 3). A connective tissue graft was carried out to cover the defect, as well as a biopsy of the lesion at the apex of implant \# 31 was performed to confirm the clinical-radiological diagnosis.

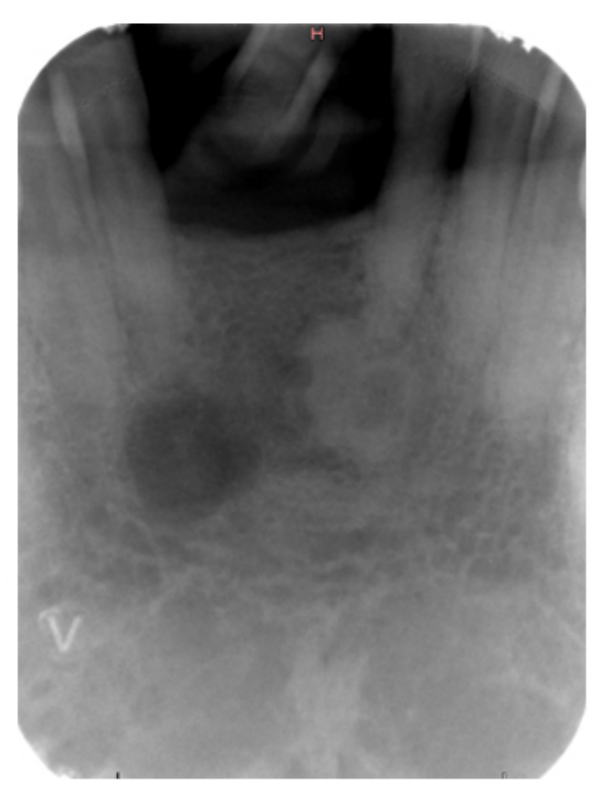

Figure 1. Initial periapical radiograph. Lesion at an osteolytic stage in the periapical region of tooth \# 42 and cementoblastic stage at the apex of tooth \# 32

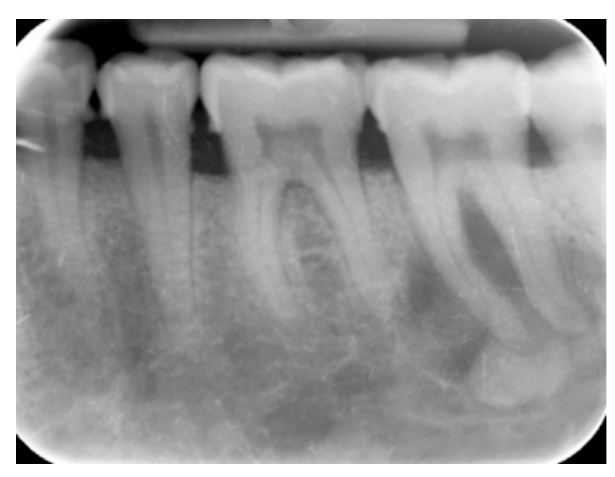

Figure 2. Periapical radiograph of quadrant 3 , showing a FCOD 
Histology examination showed fibrous cellular tissue containing a few bone trabeculae and some immature, round, cemental fragments confirming PCOD diagnosis (see Figure 4).

Periapical radiographs allowed us to follow the evolution of the lesions following the implant placement. At the 2-month follow-up, a radiograph showed maturation of both lesions. The changes in the nature of the bone tissue did not seem to affect the favorable osseointegration of the implants. After seven months, implants were loaded with single crowns (see Figure 5). At a 6-month follow-up, a periapical radiograph showed that the lesions were stable and the implants osseointegrated and functional (see Figure 6).

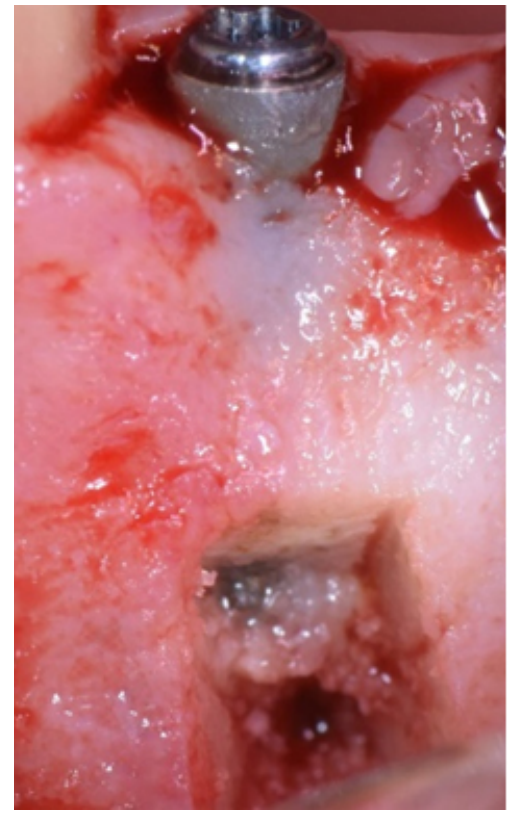

Figure 3. Photograph of implant site at four months after implant placement. Bone loss around the implant neck and the site of the biopsy is visible

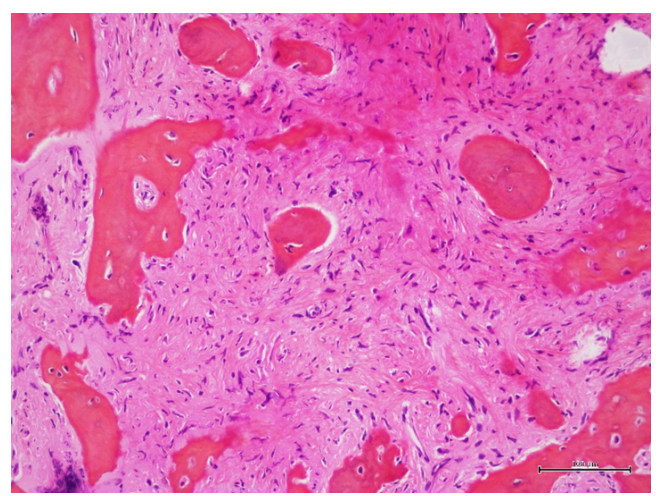

Figure 4. Histopathological section showing a fibrous cellular tissue containing a few bone trabeculae and some cemental fragments (HE stain)

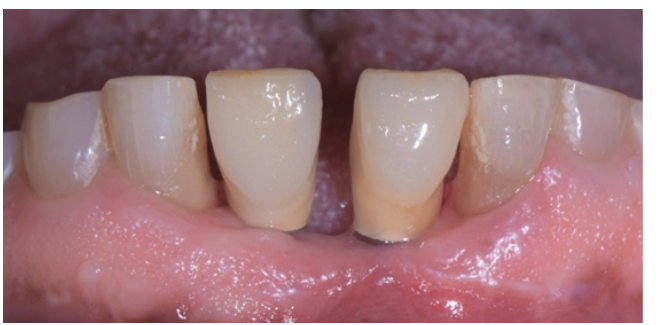

Figure 5. Clinical photograph after fixtures placement

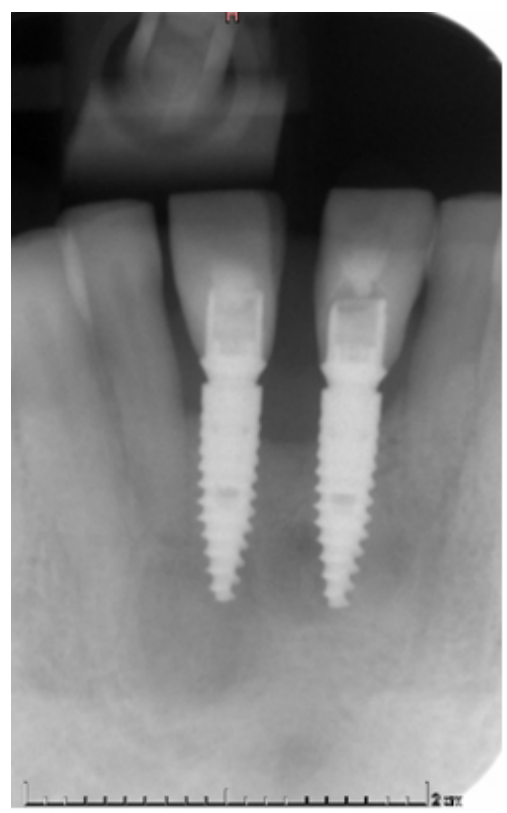

Figure 6. Periapical radiograph at six months follow-up

\section{Discussion}

The majority of CODs are totally asymptomatic, and their diagnosis is based on clinical and radiological exams. Therefore either a biopsy or treatment is not necessary. A follow-up is generally recommended in order to follow the evolution of the lesion. Many complications have been reported such as pain, ${ }^{[13-16]}$ swelling, ${ }^{[16]}$ implant failure, ${ }^{[11,15,17]}$ chronic osteomyelitis ${ }^{[11]}$ or even fractures following biopsy or resection of the lesion. ${ }^{[16]}$ The development of these complications is due to the altered bone quality of the affected area; indeed, the exposition of these avascular and acellular tissues leads to secondary infections that are difficult to control and treat. The lack of vascularization prevents antibiotics from reaching the infected site, and for this reason, a resection of the lesion is sometimes necessary.

This bone quality is not optimal to place implants, and many authors advise not to perform such procedures. For this reason, if implants placement is necessary or requested by the patient, a conservative protocol must be applied. The use of piezosurgery or sufficient cooling during drilling is rec- 
ommended to minimize trauma in order to avoid the risk of osteomyelitis, bone necrosis, and sequestrum formation.

To the best of our knowledge, no cases of implant placement in PCOD have been reported. However, a few case reports have described implant placement in jaws affected by FLCOD.

In 6 publications dealing with 6 cases, the implants were placed within or outside the lesion; ${ }^{[11,12,15,17-19]}$ only 3 cases among the 6 described were successfully integrated. ${ }^{[12,18,19]}$

Gerlach et al. ${ }^{[15]}$ described implant failures in a FLCOD, but the mandible was also affected by a cemento-ossifying fibroma (COF). Oliveira et al., ${ }^{[17]}$ as well as Shin et al., ${ }^{[11]}$ have also reported cases of implants failures in FLCOD manifested by the loose implant, swelling, and pain. Park et al., ${ }^{[12]}$ on the other hand, have demonstrated the possibility of long-term implant survivability in FCOD with implant sta-

\section{REFERENCES}

[1] Kramer IR, Pindborg JJ, Shear M. The WHO Histological Typing of Odontogenic Tumours. A commentary on the Second Edition. Cancer. 1992; 70: 2988-2994. https : //doi.org/10.1002/1097-0142(19921215)70: 12<2988: :AID-CNCR2820701242>3.0.CO;2-V

[2] El-Naggar Chan JKC, Grandis JR, Takata T, et al. WHO classification of Head and Neck Tumours. Chapter 8: Odontogenic and maxilofacial bone tumours. 4th ed. IARC: Lyon; 2017.

[3] Alsufyani NA, Lam EWN. Osseous (cemento-osseous) dysplasia of the jaws: clinical and radiographic analysis. J Can Dent Assoc. 2011; 77: b70.

[4] Brad WN, Douglas DD, Carl MA, et al. Chi - Oral and Maxillofacial Pathology-Saunders (2015). 4th ed.

[5] Eversole R, Su L, ElMofty S. Benign fibro-osseous lesions of the craniofacial complex. A review. Head Neck Pathol. 2008; 2: 177202. PMid:20614314.https ://doi .org/10.1007/s12105-008 $-0057-2$

[6] Summerlin DJ, Tomich CE. Focal cemento-osseous dysplasia: a clinicopathologic study of 221 cases. Oral Surg Oral Med Oral Pathol. 1994; 78: 611-620. https://doi.org/10.1016/0030-4220 (94 ) $90174-0$

[7] Senia ES, Sarao MS. Periapical cemento-osseous dysplasia: a case report with twelve-year follow-up and review of literature. Int Endod J. 2015; 48: 1086-1099. PMid:25425097. https ://doi.org/10.1 $111 /$ iej. 12417

[8] Kawai T, Hiranuma H, Kishino M, et al. Cemento-osseous dysplasia of the jaws in 54 Japanese patients: a radiographic study. Oral Surg Oral Med Oral Pathol Oral Radiol Endod. 1999; 87: 107-114. https://doi.org/10.1016/S1079-2104(99)70303-3

[9] Roghi M, Scapparone C, Crippa R, et al. Periapical cemento-osseous dysplasia: clinicopathological features. Anticancer Res. 2014; 34 : 2533-2536.

[10] Kato C de NA de O, de Arruda JAA, Mendes PA, et al. Infected Cemento-Osseous Dysplasia: Analysis of 66 Cases and Literature bility at 15 years. He explained it by the fact that the screw was placed within a mature lesion (late stage), increasing bone-implant contact (BIC). The implant was removed after 16 years because of peri-implantitis. A most recent publication has also reported the success of implant osseointegration in FLCOD. ${ }^{[18]}$

\section{Conclusion}

PCOD is a rather common asymptomatic bone lesion characterized by abnormal bone quality. However, implant placement is at risk of developing some complications; in particular clinical settings, it may be considered a favorable procedure. In such cases, it is important to perform an atraumatic technique. Our case showed favorable osseointegration of dental implants in PCOD.

\section{CONFlicts of InTEREST Disclosure}

The authors declare they have no conflicts of interest.
Review. Head Neck Pathol. 2020; 14: 173-182. PMid:31011984. https://doi.org/10.1007/s12105-019-01037-x

[11] Shin HS, Kim BC, Lim HJ, et al. Chronic osteomyelitis induced by the placement of dental implants on cemento-osseous dysplasia. Br J Oral Maxillofac Surg. 2019; 57: 268-270. PMid:30898454. https://doi.org/10.1016/j.bjoms.2019.01.014

[12] Park WB, Han JY, Jang JS, et al. Long-Term Implant Survivability of an Implant Having Direct Contact with Cementum-Like Tissue in a Preexisting Mandibular Intraosseous Lesion with a 16-Year Longitudinal Follow-up. Int J Periodontics Restorative Dent. 2019; 39: 895902. PMid:31613952. https://doi.org/10.11607/prd. 4432

[13] Bencharit S, Schardt-Sacco D, Zuniga JR, et al. Surgical and prosthodontic rehabilitation for a patient with aggressive florid cemento-osseous dysplasia: a clinical report. J Prosthet Dent. 2003; 90: 220-224. https://doi .org/10.1016/S0022-3913(03) 004 $31-1$

[14] Sukegawa S, Kanno T, Kawai H, et al. Surgical Treatment and Dental Implant Rehabilitation after the Resection of an Osseous Dysplasia. Journal of Hard Tissue Biology. 2016; 25: 437-441. https://doi.org/10.2485/jhtb.25.437

[15] Gerlach RC, Dixon DR, Goksel T, et al. Case presentation of florid cemento-osseous dysplasia with concomitant cemento-ossifying fibroma discovered during implant explantation. Oral Surg Oral Med Oral Pathol Oral Radiol. 2013; 115: e44-52. PMid:22858018. https://doi.org/10.1016/j.0000.2012.05.020

[16] Sarmento DJ de S, Monteiro BV de B, de Medeiros AMC, et al. Severe florid cemento-osseous dysplasia: a case report treated conservatively and literature review. Oral Maxillofac Surg. 2013; 17: 43-46. PMid:22249882. https ://doi .org/10.1007/s10006-0 12-0314-0

[17] Oliveira MTF, Cardoso SV, Silva CJ, et al. Failure of dental implants in cemento-osseous dysplasia: a critical analysis of a case. Revista de Odontologia da UNESP. 2014; 43: 223-227. https: //doi.org/10.1590/rou.2014.035 
[18] Shadid R, Kujan O. Success of dental implant osseointegration in a florid cemento-osseous dysplasia: A case report with 8-year follow-up. Clin Pract. 2020; 10: 1281. PMid:32952986. https: //doi.org/10.4081/cp.2020.1281

[19] Esfahanizadeh N, Yousefi H. Successful Implant Placement in a Case of Florid Cemento-Osseous Dysplasia: A Case Report and Litera- ture Review. J Oral Implantol. 2018; 44: 275-279. PMid:29406819. https://doi.org/10.1563/aaid-joi-D-17-00140

[20] Das BK, Das SN, Gupta A, et al. Florid cemento-osseous dysplasia. J Oral Maxillofac Pathol. 2013; 17: 150. PMid:23798858. https://doi.org/10.4103/0973-029X.110735 